\title{
artigo
}

Menezes, C.A.; Vasconcelos, R.S.

Distanciamento social, risco cardiometabólico e alteração psicossocial em crianças obesas durante pandemia do COVID-19

\section{Distanciamento social, risco cardiometabólico e alteração psicossocial em crianças obesas durante pandemia do COVID-19}

\author{
Social distancing, cardiometabolic risk and psychosocial change in obese children during COVID-19 pandemic
} Distancia social, riesgo cardiometabólico y cambio psicosocial en niños obesos durante la pandemia de COVID-19

\section{RESUMO}

Objetivos: Correlacionar os dados da relação cintura/estatura no distanciamento social decorrente do SARS-CoV-2 com o período antes da pandemia em crianças obesas e eutróficas, e comparar o comportamento psicossocial de ansiedade com hábitos de hiperfagia e beliscador das crianças obesas. Método: Estudo caso controle, realizado no Centro de Especialidades Médicas em Crianças e Adolescentes em Aracaju-Sergipe, com 30 crianças obesas e 10 eutróficas. Resultados: A relação cintura/estatura no pré e pós isolamento social nos meninos foi 0,64 e 0,74 respectivamente $(p<0,001)$ e nas meninas foi 0,72 antes do isolamento e 0,81 após ( $<<0,001)$; as crianças obesas já apresentavam comportamento de ansiedade tanto no pré isolamento $(\mathrm{OR}=5,4)$ quanto no pós $(\mathrm{OR}=9,6)$; e comportamento alimentar hiperfágico e beliscador no pré isolamento $(\mathrm{OR}=$ $7,2)$ e pós $(O R=9,2)$. Conclusão: Observou-se que o distanciamento social promoveu um incremento da obesidade central e ansiedade, refletida com hábitos hiperfágicos e beliscador.

DESCRITORES: Obesidade; Criança; Coronavírus; Ansiedade.

\section{ABSTRACT}

Objectives: To correlate the data of the waist / height ratio in the social distance resulting from SARS-CoV-2 with the period before the pandemic in obese and eutrophic children, and to compare the psychosocial behavior of anxiety with hyperphagia and pinching habits of obese children. Method: Case-control study carried out at the Center for Medical Specialties for Children and Adolescents in Aracaju-Sergipe, with 30 obese and 10 eutrophic children. Results: The waist / height ratio in the pre and post social isolation in boys was 0.64 and 0.74 respectively $(p<0.001)$ and in girls it was 0.72 before isolation and 0.81 after ( $<$ <.001); obese children were already showing anxiety behavior both in pre-isolation $(O R=5.4)$ and in post $(O R=9.6)$; and hyperphagic and pinching eating behavior in pre-isolation $(O R=7.2)$ and post $(O R=9.2)$. Conclusion: It was observed that social distance promoted an increase in central obesity and anxiety, reflected with hyperphagic and pinching habits.

DESCRIPTORS: Obesity; Child; Coronavirus; Anxiety.

\section{RESUMEN}

Objetivos: Correlacionar los datos de la relación cintura / altura en la distancia social resultante del SARS-CoV-2 con el período anterior a la pandemia en niños obesos y eutróficos, y comparar el comportamiento psicosocial de ansiedad con hiperfagia y hábitos de pellizco de obesos. niños. Método: Estudio de casos y controles realizado en el Centro de Especialidades Médicas de la Infancia y la Adolescencia de Aracaju-Sergipe, con 30 niños obesos y 10 eutróficos. Resultados: La relación cintura / talla en el pre y post aislamiento social en los niños fue de 0,64 y 0,74 respectivamente $(p<0,001)$ y en las niñas fue de 0,72 antes del aislamiento y 0,81 después ( $<<0,001$ ); los niños obesos ya mostraban un comportamiento de ansiedad tanto en el pre-aislamiento $(O R=5,4)$ como en el post $(O R=9,6)$; y comportamiento alimentario hiperfágico y de pellizcos antes del aislamiento $(O R=7,2)$ y después $(O R=9,2)$. Conclusión: Se observó que la distancia social promovió un aumento de la obesidad central y la ansiedad, reflejada en hábitos hiperfágicos y de pellizco.

DESCRIPTORES: Obesidad; Niño; Coronavirus; Ansiedad.

RECEBIDO EM: 14/01/2021 APROVADO EM: 09/02/2021 


\section{Carlos Alberto Menezes}

Professor Adjunto de Endocrinologia e Metabologia do curso de Medicina e docente do Programa de Pós-Graduação em Ciências da Saúde da Universidade Estadual de Santa Cruz (PPGCS/UESC). Ilhéus-BA. Médico Endocrinologista do Centro de Especialidades Médicas da Criança e Adolescentes (CEMCA).Aracaju-SE.

ORCID: 0000-0003-2306-6494

\section{Rayzza Santos Vasconcelos}

Enfermeira Especialista em Saúde Coletiva e em Enfermagem em Dermatologia, Discente do Programa de Pós-Graduação em Ciências da Saúde Universidade Estadual de Santa Cruz (PPGCS/UESC). Ilhéus-BA

ORCID: 0000-0001-9276-3731

\section{INTRODUÇÃO}

A obesidade infantil é um problema alarmante no Brasil e no mundo, com sérias repercussões cardiometabólicas e psicossocial, e encontra-se em torno de $10-15 \%$ segundo Taylor et al. ${ }^{1}$. A sua prevalência está aumentando por motivo das mudanças comportamentais ocorridas nas últimas décadas, em especial a alimentação inadequada e ao sedentaris$\mathrm{mo}^{2}$. Pode-se dizer que a obesidade é vista como uma doença crônica multifatorial, que envolve questões genéticas, ambientais e psicológicas, e seu tratamento deve ter uma abordagem multidisciplinar ${ }^{3,4}$.

A obesidade é caracterizada pelo acúmulo de tecido adiposo no organismo, que resulta do desequilíbrio entre o consumo alimentar e o gasto calórico, resultando em um balanço energético positivo, sendo considerada uma doença que com diversas implicações fisiológicas para o sistema endócrino-metabólico, e potencialmente significativas à manutenção da saúde 5 .

É sabido que a obesidade na faixa pediátrica já apresenta riscos de comorbidades cardiometabólicas, como: diabetes mellitus, hipertensão arterial sistêmica, dislipidemia, e também alguns casos de neoplasias, além das manifestações psicológicas, como ansiedade. Essas manifestações contribuem na adoção de hábitos alimentares hiperfágicos e beliscador, que ainda são exacerbados pelo distanciamento social obrigatório devido ao risco da pandemia novo coronavírus ${ }^{6}$.

A descoberta desse novo tipo de coronavírus, conhecido atualmente por Coronavírus da Síndrome Respiratória Aguda Grave ${ }^{2}$ (SARS- $\mathrm{CoV}-2$ ), na cidade de Wuhan na China em dezembro de 2019, causa a doença de Coronavírus 2019 (COVID-19) ${ }^{7}$. No Brasil, o primeiro caso foi relatado em 26 de fevereiro de 2020, e a indicação de distanciamento social no Brasil, teve início na segunda semana de março8.

O quadro clínico dessa doença pode variar entre assintomático até insuficiência respiratória, seus principais sintomas são: febre, fadiga, tosse seca, mialgia e dispneia; os indivíduos também podem apresentar cefaleia, escarro, hemoptise e diarreia'. O SARS-CoV-2 tem disseminação por todo o mundo em proporções pandêmicas, ressoando negativamente nas patologias relacionadas a obesidade, diabetes tipo 2, doença pulmonar obstrutiva crônica, doenças reumáticas em uso de corticoides e diversas neoplasias.

Diante das características do vírus, um novo comportamento social de isolamento está sendo adotado com o intuito de evitar a disseminação viral e sua proliferação ${ }^{10}$. Nesse sentido, sabe-se que distanciamento social atualmente é o único meio de evitar a propagação do novo coronavírus, embora tem repercutido negativamente em indivíduos portadores de outras patologias a exemplo de crianças com obesidade ${ }^{11,12}$.

Tendo em vista o distanciamento social estabelecido pelo COVID-19, o presente estudo objetivou: correlacionar os dados antropométricos da relação cintura/estatura no período de distanciamento social decorrente do SARS-CoV-2 com o período antes pandemia em um grupo de crianças obesas e eutróficas, e comparar o comportamento psicossocial de ansiedade com hábitos de hiperfagia e beliscador das crianças obesas no período pré e pós isolamento social.

\section{MÉTOdO}

Trata-se de um estudo caso controle realizado no Centro de Especialidades Médicas em Crianças e Adolescentes (CEMCA) em Aracaju-Sergipe. O CEMCA é um serviço público composto por uma equipe interdisciplinar (médicos, enfermeira, nutricionista, psicólogo e educador físico).

Participaram desse estudo 40 crianças na faixa etária de 6-8 anos, sendo 30 crianças obesas z escore de índice de massa corpórea (IMC) +2 e 10 crianças eutróficas z escore de IMC +1 . As crianças obesas foram selecionadas através do grupo de acompanhamento interdisciplinar no combate à obesidade infantil do CEMCA, e as crianças eutróficas eram parentes de primeiro grau das crianças obesas com a mesma faixa etária.

As crianças foram avaliadas por um período de 4 meses (março a junho de 2020) a nível domiciliar devido ao distanciamento social estabelecido pela Organização Mundial da Saúde para combate a pandemia do novo coronavírus. Não houve desistência ao final do estudo. Os resultados dos dois parâmetros clínicos (cintura e estatura) foram relatados pelos pais ou responsáveis no momento da consulta por via telefônica realizada pelos componentes da equipe interdisciplinar do CEMCA.

A avaliação das crianças em domicílio no período da quarentena, conforme estabelecido pela Secretaria Municipal de Saúde de Aracaju, foi realizada pelos pais ou responsáveis, que foram devidamente treinados pela equipe interdisciplinar via telefone por meio de vídeochamadas pelo aplicativo do WhatsApp, contendo as orientações explicativas.

Os pais ou responsáveis receberam uma fita métrica inelástica $(150 \mathrm{~cm})$ para aferição da estatura, e foram treinados a colocar as crianças na parede em posição anatômica com os pés juntos, dessa forma foi aferida a medição dessas crianças, sendo que após a verificação da estatura, eles colocaram a fita 
métrica no ponto médio entre o rebordo costal inferior e a maior elevação da crista ilíaca e verificaram a cintura. Neste sentido, foi avaliada a obesidade central através desse indicador indireto de comorbidade cardiometabólico, fazendo a divisão da cintura pela estatura. O ponto de corte para o risco cardiometabólico adotado para essa relação cintura/estatura é maior que $0,5 \cdot 13,14,15,16$

As demais variáveis (sexo, cor autodeclarada e idade) foram coletadas do prontuário das crianças que são acompanhadas no CEMCA. O comportamento alimentar hiperfágico e beliscador foi relatado pelos pais durante as consultas com psicólogo e esse comportamento foi confirmado após período de isolamento por via telefônica, onde os pais confirmaram maior ingesta de alimentos palatáveis (doces e gulosemas) em maior quantidade e frequência no grupo das crianças com obesidade.

Já, a ansiedade foi avaliada pelo psicólogo do CEMCA via telefone por meio da chamada de vídeo e também se utilizou o telemonitoramento/teleconsulta. A avaliação foi feita através da Revised Children's Manifest Anxiety Scale (RCMAS), conhecida pelo seu nome comercial "O que penso e sinto", que foi desenvolvida por Reynolds e Richmond em 1978. Esta escala propóe medir a ansiedade enquanto traço de personalidade, foi validada para o português, é composta por 37 itens em que o sujeito deve escolher a resposta sim ou não ${ }^{17}$.
As variáveis sexo, cor autodeclarada, idade, relação cintura/estatura, ansiedade, hábitos hiperfágicos e beliscador foram tabuladas pelo Statistical Package for Social Sciences - versão 22.0. Foi avaliada a associação bivariada e cálculo do Oddes ratio. Para a análise usou-se regressão logística binária e bruta e ajustada com $\mathrm{p}<0,05$ após o ajuste.

Este estudo foi aprovado pelo Comitê de Ética em Pesquisa da Universidade Estadual de Santa Cruz (UESC), por meio do Certificado de Apresentação para Apreciação Ética 04065412.600005526.

\section{RESULTADOS}

A tabela 1 apresenta os dados das crianças referente ao sexo, cor autodeclarada, relação cintura/estatura no grupo com obesidade no período de isolamento por 4 meses pelo novo coronavírus quando comparado aos dados anteriormente tabulados no acompanhamento desse grupo mensalmente no CEMCA, além dos dados das crianças eutróficas.

Observou-se que as crianças obesas tinham a faixa etária de 6-8 anos ( $\pm 7,2$ anos), sendo 15 meninos (50\%) e 15 meninas (50\%) pertencentes ao grupo de acompanhamento interdisciplinar no combate a obesidade infantil do CEMCA, e 10 crianças eutróficas. Quanto a cor autodeclarada, apresentou um Odds ratio $(\mathrm{OR})$ 7,4 e intervalo de confiança de 2,2-31,6 e com valor $\mathrm{p}<0,001$.
A relação cintura/estatura apresentou uma elevação considerável em quatro meses de distanciamento social devido ao COVID-19, esta informação é confirmada quando compara-se a relação cintura sobre estatura no período de pré e pós isolamento social tanto nos meninos $(0,64-0,74)$ com OR de $(4,6-7,2)$ e intervalo de confiança de $(1,84-11,8)$ e $p<0,001$, quanto nas meninas $(0,72-0,81)$ com OR $(4,6-$ $7,2)$ e intervalo de confiança de $(2,7-20,2)$ $e p<0,001$. Ainda em relação ao incremento dos valores da relação cintura/estatura foi maior nas meninas $(0,72-0,81)$ no período avaliado pós isolamento devido a pandemia do novo coronavírus, quando correlacionada aos meninos $(0,64-0,74)$. Não houve alteração da relação cintura/estatura no grupo eutrófico quando comparados os períodos pré e pós pandemia pelo novo coronavírus.

Em relação a tabela 2, ela demonstra a comparação de características psicossociais de 40 crianças obesas e eutróficas no período de distanciamento social devido ao novo coronavírus em comparação ao período de avaliação do grupo fechado no CEMCA. As crianças com obesidade já apresentam comportamento de ansiedade observado tanto no período pré isolamento com $\mathrm{OR}=5,4$ com intervalo de confiança de 3,2- 21,6 e $\mathrm{p}<0,001$, exacerbado pós isolamento com $\mathrm{OR}=9,6$ com intervalo de confiança de 5,84-11,8 e p<0,001 e comportamento ali-

Tabela 1: Características sociodemográficas e clínicas de crianças obesas e eutróficas no período de isolamento social devido ao novo coronavírus em comparação ao período de avaliação do grupo fechado no CEMCA, Aracaju- 2020.

\begin{tabular}{|c|c|c|c|c|c|c|}
\hline \multirow{2}{*}{ CARACTERÍSTICAS } & & \multicolumn{2}{|c|}{ \% CLASSIFICAÇÃO } & \multirow{2}{*}{ ODDS RATIO } & \multirow{2}{*}{ IC95\% } & \multirow{2}{*}{$\mathbf{p a}$} \\
\hline & & OBESO & EUTRÓFICO & & & \\
\hline \multirow{2}{*}{ Sexo } & Masculino & 15 & 05 & \multirow{2}{*}{1,1} & \multirow{2}{*}{$0,5-2,6$} & \multirow{2}{*}{0,794} \\
\hline & Feminino & 15 & 05 & & & \\
\hline \multirow{2}{*}{ Cor autodeclarada } & Preto & 20 & 08 & \multirow{2}{*}{7,4} & \multirow{2}{*}{$2,2-31,6$} & \multirow{2}{*}{$<0,001$} \\
\hline & Branco & 10 & 02 & & & \\
\hline \multirow{2}{*}{ Relação Cintura/Estatura } & Meninos & 0,64 & 0,40 & \multirow{2}{*}{4,6} & \multirow{2}{*}{$1,84-11,8$} & \multirow{2}{*}{$<0,001$} \\
\hline & Meninas & 0,72 & 0,30 & & & \\
\hline \multirow{2}{*}{$\begin{array}{l}\text { Relação Cintura/Estatura após } 4 \text { meses de isola- } \\
\text { mento pelo novo coronavírus. }\end{array}$} & Meninos & 0,74 & 0,41 & \multirow{2}{*}{7,2} & \multirow{2}{*}{$2,7-20,2$} & \multirow{2}{*}{$<0,001$} \\
\hline & Meninas & 0,81 & 0,31 & & & \\
\hline
\end{tabular}


mentar hiperfágico e beliscador no período pré isolamento com $\mathrm{OR}=7,2$ e intervalo de confiança de 2,7-20,2 complicado no período pós confinamento social pelo novo coronavírus com $\mathrm{OR}=9$, 2 e intervalo de confiança de 5,7-22,2 e p<0,001.

Por outro lado, o grupo eutrófico de crianças não apresentou relatos de ansiedade nem comportamento alimentar hiperfágico e beliscador.

\section{DISCUSSÃO}

No que abrange aos aspectos sociodemográficos, já foi relatado que a obesidade possui fator hereditário e que adultos negros possuem maior probabilidade de serem obeso, isso se confirmou para as crianças do presente estudo, na qual a cor autodeclarada preta foi predominante. Este achado está congruente com os dados publicado por Bell e Thorpe ${ }^{18}$ e por Lew et a $1^{19}$.

Porém, o resultado difere do estudo de Moretto et al.20 com 885 idosos brasileiros no qual a obesidade não se associou com a cor autodeclarada preta, mas sim com variáveis socioeconômicas como escolaridade e renda familiar, mas estas variáveis podem estar relacionadas com a cor segundo relatos de Hruby e $\mathrm{Hu}^{21}$ e de Fradkin et al. ${ }^{22}$.

Quanto aos dados antropométricos, notou-se que neste estudo as crianças apre- sentaram uma elevação considerável em três meses de distanciamento social devido ao COVID-19. Esse resultado está de acordo com o conteúdo publicado por Ashwell et al. ${ }^{23}$, pois eles concluíram que se deve manter a circunferência da cintura menor que a metade da altura, essa explicação pode ser útil para saúde pública. $\mathrm{O}$ incremento da obesidade central se deve, provavelmente, ao comportamento sedentário oriundo de maior ansiedade com hábitos hiperfágicos exacerbados nesse período ${ }^{24}$.

A obesidade na infância é um fator de risco para o desenvolvimento de doenças cardiovasculares quando adultos ${ }^{25}$. Além de causar efeitos cognitivos, ela também promove alterações hemodinâmicas ao estimular os sistemas simpático e renina-angiotensina-aldosterona, induzindo a maior concentração e retenção de soluções salinas, sobrecarregando de modo significativo o sistema cardiovascular ${ }^{26}$.

Nesse estudo, verificou-se que as crianças apresentaram ansiedade no período antes pandemia que foi exacerbada no período pós pandêmico devido confinamento social, associado a redução de atividades de gasto energético ${ }^{27}$. Com a pandemia do COVID-19 é comum a presença de sentimentos e emoções negativas, como medo, tristeza, ansiedade, aflição e estresse; por conta da ansiedade e do estresse, os indivíduos desen- volvem respostas involuntárias automáticas e também voluntárias ${ }^{28}$.

Segundo Rajkumar ${ }^{29}$, o novo coronavírus SARS-CoV-2 trouxe impacto sobre a saúde e o bem-estar das pessoas, inclusive das crianças, podendo gerar até uma verdadeira crise de saúde mental, culminando em mudanças comportamentais. O distanciamento social imposto pelo COVID-19 predispõem o consumo de alimentados industrializados e fast-food deliverys e ausência de atividades para perder peso em academias ${ }^{30}$.

Esses dados corroboram com a pesquisa de Carson et al. ${ }^{31}$, que foi demonstrado em uma revisão sistemática que o comportamento sedentário devido a maior frequência e tempo gastos em TV e jogos eletrônicos foram associados a exacerbação de comportamento de ansiedade com reflexo de hiperfagia e hábitos beliscadores.

A ansiedade é um estado emocional complexo gerado por algum estimulo estressor ${ }^{32}$. O comportamento ansioso interfere na vida das pessoas, pode ser expressado através das manifestações fisiológicas (dispneia, tensão muscular, aumento dos batimentos cardíacos, entre outros) e das cognitivas (dificuldade de concentração, pensamentos repetitivos e intrusivos desesperança, dificuldade de lembrar as informações, etc.) $)^{33}$. Deste modo, as crianças com ansiedade apresentam mudanças comportamentais e alimentares,

Tabela 2: Características psicossociais de crianças obesas e eutróficas no período de isolamento social devido ao novo coronavírus em comparação ao período de avaliação do grupo fechado no CEMCA, Aracaju-2020.

\begin{tabular}{|c|c|c|c|c|c|c|}
\hline \multirow{2}{*}{ CARACTERÍSTICAS } & & \multicolumn{2}{|c|}{ \% CLASSIFICAÇÃO } & \multirow{2}{*}{ ODDS RATIO } & \multirow{2}{*}{ IC95\% } & \multirow{2}{*}{$\mathbf{p a}$} \\
\hline & & OBESO & EUTRÓFICO & & & \\
\hline \multirow{2}{*}{ Sexo } & Masculino & 15 & 05 & \multirow{2}{*}{1.1} & \multirow{2}{*}{$0,5-2,6$} & \multirow{2}{*}{0,794} \\
\hline & Feminino & 15 & 05 & & & \\
\hline \multirow{2}{*}{ Ansiedade pela obesidade pré coronavírus } & $\operatorname{Sim}$ & 24 & - & \multirow{2}{*}{5,4} & \multirow{2}{*}{$3,2-21,6$} & \multirow{2}{*}{$<0,001$} \\
\hline & Não & 06 & - & & & \\
\hline \multirow{2}{*}{ Ansiedade pela obesidade pós coronavírus } & $\operatorname{Sim}$ & 29 & - & \multirow{2}{*}{9,6} & \multirow{2}{*}{$5,84-11,8$} & \multirow{2}{*}{$<0,001$} \\
\hline & Não & 01 & 10 & & & \\
\hline \multirow{2}{*}{$\begin{array}{l}\text { Comportamento alimentar hiperfágico e belisca- } \\
\text { dor pré coronavírus }\end{array}$} & Sim & 25 & - & \multirow{2}{*}{7,2} & \multirow{2}{*}{$2,7-20,2$} & \multirow{2}{*}{$<0,001$} \\
\hline & Não & 05 & - & & & \\
\hline \multirow{2}{*}{$\begin{array}{l}\text { Comportamento alimentar hiperfágico e belisca- } \\
\text { dor pós coronavírus }\end{array}$} & $\operatorname{Sim}$ & 29 & - & \multirow{2}{*}{9,2} & \multirow{2}{*}{$5,7-22,2$} & \multirow{2}{*}{$<0,001$} \\
\hline & Não & 01 & - & & & \\
\hline
\end{tabular}


adotando hábitos alimentares compulsivos e com frequência.

A obesidade na faixa etária pediátrica está relacionada a relatos de depressão, ansiedade, solidão e mudanças comportamentais. Estes podem interferir na vida da criança, prejudicando o desempenho escolar e as relações familiares e sociais. Segundo Rodrigues, Pombo e Koifman ${ }^{34}$, o excesso de adipocinas, na infância, atua delongando de forma progressiva o desenvolvimento neuropsicomotor e cognitivo da criança, visto que a multiplicação dos adipócitos promove um retardamento global no desenvolvimento neurológico com implicações no sistema imunológico, tornando a criança obesa mais vulnerável a doenças mentais e infectocontagiosas, além de aumentam as chances de obesidade na adultez.
Diante do cenário atual relacionado a pandemia do COVID-19, percebe-se que ela leva a adoção de comportamentos não saudáveis, como dieta de baixa qualidade e falta de exercício físico, além disso ainda contribui para agravar aspectos emocionais da pessoa ${ }^{35}$. Por conta disso, observa-se que as crianças obesas que participaram desse estudo apresentaram ansiedade e aumento da relação cintura/estatura.

O estudo aponta para a necessidade dos profissionais de saúde utilizarem a relação cintura/estatura, já que é um bom indicador de obesidade central, pela sua praticidade de execução e pelos dados objetivos que essa relação fornece mesmo na faixa etária mais precoce, pois a partir desse indicador pode-se intervir de maneira enérgica nesse grupo pediátrico no intuito de evitar comorbidades cardiometabólicas o mais precoce possível.

\section{CONCLUSÃO}

Diante do exposto, observou-se que o distanciamento social promoveu um incremento da obesidade central determinado pela relação cintura/estatura, e também da ansiedade. A ansiedade refletida com hábitos hiperfágicos e beliscador é uma realidade das crianças com obesidade e que o confinamento social determinado pela pandemia fez exacerbar esse comportamento psicossocial. Deste jeito, deve-se atuar de maneira eficaz estimulando o gasto energético e orientação psicossocial individualiza$\mathrm{da}$, familiar e grupal para amenizar essas repercussóes psicológica para esse grupo com grande fragilidade social.

\section{REFERÊNCIAS}

1. Taylor VH, Forhan M, Vigod SN, McIntyre RS, Morrison KM. The impact of obesity on quality of life. Best Pract Res Clin Endocrinol Metab [Internet]. 2013[cited 2020 apr. 11];27(2):139-46. Available at: https://pubmed.ncbi.nlm.nih.gov/23731876/

2. Ferreira AP de S, Szwarcwald CL, Damacena GN. Prevalence of obesity and associated factors in the Brazilian population: a study of data from the 2013 National Health Survey. Rev Bras Epidemiol [Internet]. 2019[cited 2020 may 03];22:e190024. Available at: http://www.scielo.br/scielo.php?script=sci_arttext\&pid=S1415-790X2019000100420\&Ing=en\&nrm=iso\&tlng=pt

3. Frontzekl LGM, Bernardes LR, Modena CM. Obesidade infantil: compreender para melhor intervir. Rev abordagem gestalt [Internet]. 2017[cited 2020 may 03];23(2):167-74. Available at: http://pepsic.bvsalud.org/scielo.php?script=sci_arttext\&pid=S1809-68672017000200005

4. Menezes,CA, Loureiro, ZJP, Vasconcelos, RS. Clinical,dietary and psychosocial effectiveness of interdisciplinary care in child and juvenile obesity. Journal of Pediatrics and Infants. 2020:3(2). https:// www.innovationinfo.org/articles/JPI/JPI-124.pdf.

5. Lima RCA, Carnevali Júnior LC, Ferreira LLR, Bezerra LTL, Bezerra $\pi \mathrm{T}$, Lima B da C. Principais alterações fisiológicas decorrentes da obesidade: um estudo teórico. SANARE - Rev Políticas Públicas [Internet]. 2018 [cited 2020 apr. 08];17(2):56-65. Available at: https:// sanare.emnuvens.com.br/sanare/article/view/1262

6. Lindberg L, Hagman E, Danielsson P, Marcus C, Persson M. Anxiety and depression in children and adolescents with obesity: A nationwide study in Sweden. BMC Med [Internet]. 2020 [cited 2020 apr. 08];18(30):1-9. Available at: https://pubmed.ncbi.nlm.nih. gov/32079538/
7. Lourenção LG. A Covid-19 e os desafios para o sistema e os profissionais de saúde. Enferm em Foco [Internet]. 2020 [cited 2020 june 30];11(1):6-7. Available at: http://revista.cofen.gov.br/ index.php/enfermagem/article/view/3488

8. Lobo A de P, Cardoso-dos-Santos AC, Rocha MS, Pinheiro RS, Bremm JM, Macário EM, et al. COVID-19 epidemic in Brazil: Where are we at? Int J Infect Dis [Internet]. 2020 [cited 2020 june 26];97:382-385. Available at: https://doi.org/10.1016/j. ijid.2020.06.044

9. He F, Deng Y, Li W. Coronavirus disease 2019: What we know? J Med Virol [Internet]. 2020 [cited 2020 may 03];92(7):719-25. Available at: https://onlinelibrary.wiley.com/doi/abs/10.1002/ jmv.25766

10. Huang C, Wang Y, Li X, Ren L, Zhao J, Hu Y, et al. Clinical features of patients infected with 2019 novel coronavirus in Wuhan, China. Lancet [Internet]. 2020 [cited 2020 apr. 04];395(10223):497-506. Available at: https://isaric.tghn.org/protocols/

11. Zimmermann $P$, Curtis N. Coronavirus infections in children including COVID-19. Pediatr Infect Dis J [Internet]. 2020 [cited 2020 apr. 14];39(5):355-68. Available at: https://journals.lww. com/10.1097/INF.0000000000002660

12. World Health Organization. Coronavirus disease (COVID 19) Pandemic [Internet]. Geneva: Who, 2020 [cited 2020 apr. 14]. Available at: https://www.who.int/emergencies/diseases/ novel-coronavirus-2019?gclid=Cj0KCQjw6PD3BRDPARIsAN8pHuG6kcTY-SjLluVu9jHuCekYPvSqqf_OonwsSi34WrDv074likns05MaAsagEALw_wcB

13. Sacher PM, Kolotourou M, Chadwick PM, Cole TJ, Lawson MS, Lucas $A$, et al. Randomized controlled trial of the MEND program: $A$ family-based community intervention for childhood obesty. Obesity 
[Internet]. 2010 [cited 2020 apr. 27];18(suppl. 1):s62-8. Available at: https://pubmed.ncbi.nlm.nih.gov/20107463/

14. Yoo EG. Waist-to-height ratio as a screening tool for obesity and cardiometabolic risk. Korean J Pediatr [Internet]. 2016 [cited 2020 apr. 06];59(11):425-31. Available at: /pmc/articles/ PMC5118501/?report=abstract

15. Yan W, Bingxian H, Hua Y, Jianghong D, Jun C, Dongliang G, et al. Waist-to-Height Ratio is an Accurate and Easier Index for Evaluating Obesity in Children and Adolescents*. Obesity [Internet]. 2007 [cited 2020 may 02];15(3):748-52. Available at: http://doi.wiley. com/10.1038/oby.2007.601

16. Magalhães El da S, Sant'Ana LF da R, Priore SE, Franceschini $\mathrm{S}$ do CC. Perímetro da cintura, relação cintura/estatura e perímetro do pescoço como parâmetros na avaliação da obesidade central em crianças. Rev Paul Pediatr [Internet]. 2014 [cited 2020 apr. 08];32(3):273-81. Available at: http://www.scielo.br/scielo. php?script=sci_arttext\&pid=S0103-05822014000300273\&l$\mathrm{ng}=\mathrm{en} \& \mathrm{nrm}=\mathrm{iso}$

17. Gorayeb MA, Gorayeb R. "O que penso e sinto"- adaptação da Revised Children's Manifest Anxiety Scale (RCMAS) para o português. Temas Psicol [Internet]. 2008 [cited 2020 june 26];16(1):3545. Available at: http://pepsic.bvsalud.org/scielo.php?script=sci_ arttext\&pid=S1413-389X2008000100003

18. Bell CN, Thorpe RJ. Income and Marital Status Interact on Obesity Among Black and White Men. Am J Mens Health [Internet]. 2019 [cited 2020 may 04];13(1):1-9. Available at: https://pubmed. ncbi.nlm.nih.gov/30767595/

19. Lew KN, Dorsen C, Melkus GD, Maclean M. Prevalence of obesity, prediabetes, and diabetes in sexual minority women of diverse races/ethnicities: findings from the 2014-2015 BRFSS surveys. Diabetes Educ [Internet]. 2018 [cited 2020 may 16];44(4):348-60. Available at: https://pubmed.ncbi.nlm.nih.gov/29808733/

20. Moretto MC, Fontaine AM, Garcia C de AMS, Neri AL, Guariento ME. Association between race, obesity and diabetes in elderly community dwellers: data from the FIBRA study. Cad Saude Publica [Internet]. 2016 [cited 2020 may 22];32(10):e00081315. Available at: http://dx.doi.org/10.1590/0102-311X00081315

21. Hruby A, Hu FB. The epidemiology of obesity: a big picture. Pharmacoeconomics [Internet]. 2015 [cited 2020 june 27];33(7):67389. Available at: /pmc/articles/PMC4859313/?report=abstract

22. Fradkin C, Valentini NC, Nobre GC, dos Santos JOL. Obesity and overweight among brazilian early adolescents: variability across region, socioeconomic status, and gender. Front Pediatr [Internet]. 2018 [cited 2020 apr. 24];6(81):1-8. Available at: /pmc/articles/ PMC5897517/?report=abstract

23. Ashwell M, Hsieh SD. Six reasons why the waist-to-height ratio is a rapid and effective global indicator for health risks of obesity and how its use could simplify the international public health message on obesity. Int J Food Sci Nutr [Internet]. 2005 [cited 2020 apr. 10];56(5):303-7. Available at: https://pubmed.ncbi.nlm.nih. gov/16236591/

24. Wadolowska L, Hamulka J, Kowalkowska J, Kostecka M,
Wadolowska K, Biezanowska-Kopec R, et al. Prudent-active and fast-food-sedentary dietary-lifestyle patterns: The association with adiposity, nutrition knowledge and sociodemographic factors in Polish teenagers-The ABC of healthy eating project. Nutrients [Internet]. 2018 [cited 2020 may 28];10(12):1-18. Available at: / pmc/articles/PMC6315952/?report=abstract

25. Pelegrini A, Silva DAS, Silva JMFDL, Grigollo L, Petroski EL. Anthropometric indicators of obesity in the prediction of high body fat in adolescents. Rev Paul Pediatr [Internet]. 2015 [cited 2020 may 10];33(1):56-62. Available at: http://dx.doi.org/10.1016/j. rpped.2014.06.007

26. Costa MC, Brito LL, Barbosa PJB, Lessa I. Adiponectin and low cardiometabolic risk in obese women. Arq Bras Endocrinol Metabol [Internet]. 2011 [cited 2020 apr. 28];55(2):146-54. Available at: http://www.scielo.br/scielo.php?script=sci_arttext\&pid=S0004-27302011000200007\&lng=en\&nrm=iso\&tlng=pt

27. Hughes AR, Farewell K, Harris D, Reilly JJ. Quality of life in a clinical sample of obese children. Int J Obes [Internet]. 2007 [cited 2020 apr. 11];31(1):39-44. Available at: www.nature.com/ijo

28. Weide JN, Vicentini ECC, Araujo MF de, Machado W de L, Enumo SRF. Cartilha para enfrentamento do estresse em tempos de pandemia. PUC-Campinas. Porto Alegre; 2020.

29. Rajkumar RP. COVID-19 and mental health: A review of the existing literature. Asian J Psychiatr [Internet]. 2020 [cited 2020 may 02];52:102066. Available at: /pmc/articles/PMC7151415/?report=abstract

30. Sociedade Brasileira de Pediatria. Obesidade em crianças e adolescentes e COVID-19 [Internet]. Pediatria SBP: 2020 [cited 2020 may 04]. Available at: https://www.sbp.com.br/fileadmin/user_upload/22443c-NA_-_Obesid_em_Crianc_Adolesc_e_COVID-19_. pdf

31. Carson V, Hunter S, Kuzik N, Gray CE, Poitras VJ, Chaput JP, et al. Systematic review of sedentary behaviour and health indicators in school-aged children and youth: An update. Appl Physiol Nutr Metab [Internet]. 2016 [cited 2020 apr. 07];41(6):S240-65. Available at: https://pubmed.ncbi.nlm.nih.gov/27306432/

32. Menezes AK da S, Moura LF de, Mafra VR. Transtorno de ansiedade generalizada: uma revisão da literatura e dados epidemiológicos. Amaz Sci Heal [Internet]. 2017 [cited 2020 may 02];5(3):42-9. Available at: http://ojs.unirg.edu.br/index.php/2/article/view/1323

33. Batista MA, Oliveira SM da SS. Sintomas de ansiedade mais comuns em adolescentes. Psic [Internet]. 2005 [cited 2020 may 03];6(2):43-50. Available at: http://pepsic.bvsalud.org/pdf/psic/ v6n2/v6n2a06.pdf

34. Rodrigues LG, Pombo N, Koifman S. Prevalence of metabolic changes in children and adolescents: a systematic review. Rev Paul Pediatr [Internet]. 2011[cited 2020 may 03];29(2):277-88. Available from: https://www.scielo.br/pdf/rpp/v29n2/a21v29n2.pdf

35. Balanzá-Martínez V, Atienza-Carbonell B, Kapczinski F, Boni RB De. Lifestyle behaviours during the COVID 19 - time to connect. Acta Psychiatr Scand [Internet]. 2020 [cited 2020 may 04];141(5):399400. Available at: https://doi.org/10.1016/S2468-2667 